\title{
TWO-VIEW MEDIAN CORRELATION ANALYSIS
}

\author{
Yanmin Zhu ${ }^{\mathrm{a}}$, Shuzhi Su${ }^{\mathrm{b}^{*}}$, Gaoming Yang ${ }^{\mathrm{b}}$, Bin Ge $\mathrm{Ge}^{\mathrm{b}}$ \\ and Ping Zheng ${ }^{b}$ \\ aAnhui University of Science and Technology, Huainan, Anhui, 232001, \\ P. R. China \\ ${ }^{\mathrm{b}}$ College of Computer Science and Engineering, Anhui University of Science and \\ Technology, Huainan, Anhui, 232001, P. R. China
}

\begin{abstract}
Canonical correlation analysis based on supervised information is able to learn discriminant correlation features from two-view data, which plays an important role in pattern recognition and machine learning. However, such methods mainly employ class means that are sensitive to outlier data. To solve the issue, we propose a robust two-view feature learning method, called two-view median correlation analysis. In the method, a discriminant median scatter of each view is constructed in order to enhance the robustness of outlier data, and we learn correlation features with well class separability by further constraining the discriminant median scatters on the basis of maximum between-view correlation. Promising experiment results have demonstrated the effectiveness of our method.
\end{abstract}

Keywords: multi-view feature learning, canonical correlation analysis, supervised information, image recognition.

\footnotetext{
${ }^{*}$ Corresponding author.

E-mail address: sushuzhi@foxmail.com (Shuzhi Su).
}

Copyright (C) 2018 Scientific Advances Publishers 2010 Mathematics Subject Classification: 68W25.

Submitted by Jianqiang Gao.

Received September 8, 2018 


\section{Introduction}

Multi-view feature learning is a hot research subject in pattern recognition and machine learning. Canonical correlation analysis (CCA) plays an important role in multi-view feature learning. The method can learn correlation features from two-view data by maximizing the correlation between two-view data. Up to now, CCA has been applied into many real-world applications, such as emotion analysis [1] and fMRI data processing [2]. As an unsupervised method, CCA is difficult to utilize class labels that can further enhance the class separability of correlation features. Aiming at this issue, Sun et al. [3] maximize the intra-class correlation of different views and simultaneously minimize the inter-class correlation of different views, and further proposed a discriminant CCA method. Additionally, generalized CCA [4] was proposed by considering intra-class constraints on the basis of the maximum of between-view correlation. From different viewpoints, intraview and inter-view supervised correlation analysis [5] exploits supervised correlation information both within-view data and betweenview data.

Most of supervised CCA-related methods are based on class means that are sensitive to outlier data. To solve the problem, we propose a novel robust feature learning method, called two-view median correlation analysis (TMCA). In the method, we construct a discriminant median scatter of each view, and the scatter can effectively enhance the robustness for outlier data. Additionally, our method also gives a novel supervised correlation analysis model that maximizes the between-view correlation and simultaneously minimizes the discriminant median scatters. On two face image datasets, we design some experiments in order to estimate our method, and extensive experimental results have demonstrated the effectiveness of our method. 


\section{Review of CCA}

Suppose that $X=\left[x_{1}, x_{2}, \ldots, x_{n}\right] \in R^{d_{x} \times n}$ and $Y=\left[y_{1}, y_{2}, \ldots, y_{n}\right]$ $\in R^{d_{y} \times n}$ are two view datasets corresponding to the $n$ objects, where $d_{x}$ and $d_{y}$ denote the dimension of view datasets. $\left\{x_{i}, y_{i}\right\}(i=1,2, \ldots, n)$ correspond to the same object. CCA aims at optimizing correlation projection directions $\alpha \in R^{d_{x} \times 1}$ and $\beta \in R^{d_{x} \times 1}$ so that projected lowdimension data (i.e., correlation features) $\alpha^{T} X$ and $\beta^{T} Y$ possess the maximal between-view correlation. More specifically, $\alpha$ and $\beta$ can be obtained by solving the following optimization problem [6]:

$$
\begin{aligned}
\max _{\alpha, \beta} & \frac{1}{n} \sum_{i=1}^{n} \alpha^{T}\left(x_{i}-\bar{x}\right)\left(y_{i}-\bar{y}\right)^{T} \beta \\
\text { s.t. } & \frac{1}{n} \sum_{i=1}^{n} \alpha^{T}\left(x_{i}-\bar{x}\right)\left(x_{i}-\bar{x}\right)^{T} \alpha=1 \\
& \frac{1}{n} \sum_{i=1}^{n} \beta^{T}\left(y_{i}-\bar{y}\right)\left(y_{i}-\bar{y}\right)^{T} \beta=1,
\end{aligned}
$$

where $\bar{x}$ and $\bar{y}$ are the global means, i.e., $\bar{x}=\frac{1}{n} \sum_{i=1}^{n} x_{i}$ and $\bar{y}=\frac{1}{n} \sum_{i=1}^{n} y_{i}$

\section{Two-View Median Correlation Analysis}

Since CCA is unsupervised, it is difficult to utilize class labels. Up to now, some supervised CCA-related methods have been proposed by means of class means, but these methods are sensitive to outlier data. To solve the problem, we propose a novel TMCA method. Next, we describe our method in detail. In TMCA, we first construct the discriminant median scatter of correlation features in each view: 


$$
\left\{\begin{array}{l}
\alpha^{T} R_{x} \alpha=\frac{1}{n} \sum_{i=1}^{n} \alpha^{T}\left(x_{i}-\hat{x}_{i}\right)\left(x_{i}-\hat{x}_{i}\right)^{T} \alpha, \\
\beta^{T} R_{y} \beta=\frac{1}{n} \sum_{i=1}^{n} \beta^{T}\left(y_{i}-\hat{y}_{i}\right)\left(y_{i}-\hat{y}_{i}\right)^{T} \beta,
\end{array}\right.
$$

where $\hat{x}_{i}$ ( or $\left.\hat{y}_{i}\right)$ defines the class median vector of all the samples that have the same class label as $x_{i}$ (or $y_{i}$ ) [7]. Different from intra-class scatters based on class means, the discriminant median scatter not only is more robust to outliers but also can preserve useful information hidden in raw data. On the basis of the discriminant median scatters, we further present a supervised correlation analysis model:

$$
\begin{array}{lc}
\max _{\alpha, \beta} & \alpha^{T} S_{x y} \beta \\
\text { s.t. } & \alpha^{T} R_{x} \alpha=1, \quad \beta^{T} R_{y} \beta=1,
\end{array}
$$

where $S_{x y}=\frac{1}{n} \sum_{i=1}^{n}\left(x_{i}-\bar{x}\right)\left(y_{i}-\bar{y}\right)^{T}$. The model maximizes the between-view correlation and at the same time minimizes the discriminant median scatter of each view. Thus, besides correlation advantages of CCA, correlation features from TMCA further possess well class separability and outlier robustness.

With the help of Lagrange multiplier approach [4], Equation (3) can be equivalently translated into

$$
\left\{\begin{array}{l}
S_{x y} R_{y}^{-1} S_{y x} \alpha=\lambda^{2} R_{x} \alpha \\
S_{y x} R_{x}^{-1} S_{x y} \beta=\lambda^{2} R_{y} \beta
\end{array}\right.
$$

where $\lambda$ is the generalized eigenvalue and $S_{y x}$ denotes the transport matrix of $S_{x y}$, i.e., $S_{y x}=S_{x y}^{T}$. By solving Equation (4), we can obtain the top dpairs of correlation projection directions $\left\{\alpha_{i}, \beta_{i}\right\}_{i=1}^{d}$ corresponding to the first $d$ largest generalized eigenvalues. Then, we can construct correlation 
projection matrices $A=\left[\alpha_{1}, \alpha_{2}, \ldots, \alpha_{d}\right]$ and $B=\left[\beta_{1}, \beta_{2}, \ldots, \beta_{d}\right]$, and correlation features $\tilde{x}_{i}$ and $\tilde{y}_{i}$ of $x_{i}$ and $y_{i}(i=1,2, \ldots, n)$ can be extracted in the form of $\tilde{x}_{i}=A^{T} x_{i}$ and $\widetilde{y}_{i}=B^{T} y_{i}$.

\section{Experimental Results and Analysis}

To estimate our method, we design some experiments on two face image datasets, i.e., AT \& $\mathrm{T}$ dataset [8] and $\mathrm{AR}$ dataset [9], and the datasets belong to single-view image dataset. As in [10], we employ Coiflets and Daubechies wavelets to extract two low-frequency subimages (i.e., two view data) of each image, and then the dimensionality of each view data is reduced to 100 by means of principal component analysis. In the experiments, our method is compared with discriminant CCA (DCCA) [3] and CCA. For all the methods, we utilize the nearest neighbour classifier in final recognition tasks, and each method reports the best recognition rates on all possible dimensions.

On the two datasets, we randomly choose $q(q=4,5,6,7,8)$ images per individual for training, and the rest images can be treated as testing images. We repeatedly run every random experiment for ten times, and Tables 1 and 2 exhibit the average recognition rates. Compared to CCA, DCCA further considers class labels by the maximum intra-class correlation and the minimum inter-class correlation of between-view data. Thus correlation features from DCCA possess better class separability than those from CCA. In Tables 1 and 2, it can be also seen that DCCA has the higher recognition rates than CCA. Different from DCCA, our method constrains the discriminant median scatter of each view on the basis of the between-view correlation, and the scatter is more robust for outlier data, which are important reasons why our method is superior to DCCA. From Tables 1 and 2, we can observe that our method always possesses the best recognition performance. All the experimental results in this section can give a reasonable observation that our method is an effective method for recognition tasks. 
Table 1. The average recognition rates (\%) on the AT\&T dataset

\begin{tabular}{cccccc}
\hline & 4 Train & 5 Train & 6 Train & 7 Train & 8 Train \\
\hline TMCA & 93.25 & 95.60 & 97.69 & 98.08 & 99.50 \\
DCCA & 88.71 & 93.80 & 95.50 & 96.75 & 99.38 \\
CCA & 45.86 & 78.38 & 90.35 & 93.19 & 93.83 \\
\hline
\end{tabular}

Table 2. The average recognition rates (\%) on the AR dataset

\begin{tabular}{cccccc}
\hline & 4 Train & 5 Train & 6 Train & 7 Train & 8 Train \\
\hline TMCA & 97.36 & 98.69 & 99.00 & 99.29 & 99.35 \\
DCCA & 96.79 & 98.31 & 98.72 & 99.14 & 99.15 \\
CCA & 95.58 & 97.09 & 97.86 & 98.46 & 98.58 \\
\hline
\end{tabular}

\section{Conclusion}

How to learn discriminant correlation features from two-view data is an important research topic under the correlation analysis framework. Up to now, some supervised CCA-related methods have been proposed, but these methods are mainly based on class means that are sensitive to outlier data. Therefore, we propose the TMCA method. Our method constructs the discriminant median scatter for each view, which is robust to outlier data and can preserve useful information hidden in raw data. Besides the correlation advantages inherited from CCA, our method has well class separability and outlier robustness by maximizing the correlation between two views and simultaneously minimizing the discriminant median scatter of each view. Experimental results on the real-world face image datasets have showed that our method is effective for recognition tasks.

\section{Acknowledgements}

This work is supported by the National Natural Science Foundation of China (Grant Nos. 61806006, 61572034 and 61240023), the Natural Science Research Project of Colleges and Universities in Anhui Province 
(Grant No. KJ2018A0083), Anhui Natural Science Foundation (Grant

No.1808085MG221), and Youth Science Research Project of Anhui

University of Science \& Technology (Grant No.QN2017208).

\section{References}

[1] K. Hong, G. Liu, W. Chen and Sheng Hong, Classification of the emotional stress and physical stress using signal magnification and canonical correlation analysis, Pattern Recognition 77 (2018), 140-149.

DOI: https://doi.org/10.1016/j.patcog.2017.12.013

[2] Z. Yang, X. Zhuang, K. Sreenivasan, V. Mishra, T. Curran, R. Byrd, R. Nandy and D. Cordes, 3D spatially-adaptive canonical correlation analysis: Local and global methods, NeuroImage 169 (2018), 240-255.

DOI: https://doi.org/10.1016/j.neuroimage.2017.12.025

[3] T. Sun, S. Chen, J. Yang and Pengfei Shi, A supervised combined feature extraction method for recognition, Proceedings of the IEEE International Conference on Data Mining (2008), 1043-1048.

[4] Q. S. Sun, Z. D. Liu, P. A. Heng and De-Sen Xia, A theorem on the generalized canonical projective vectors, Pattern Recognition 38(3) (2005), 449-452.

DOI: https://doi.org/10.1016/j.patcog.2004.08.009

[5] X. Y. Jing, R. Hu, Y. P. Zhu, Shanshan Wu, Chao Liang and Jingyu Yang, Intra-view and inter-view supervised correlation analysis for multi-view feature learning, Proceedings of the AAAI Conference on Artificial Intelligence 14 (2014), 1882-1889.

[6] C. O. Sakar and O. Kursun, Discriminative feature extraction by a neural implementation of canonical correlation analysis, IEEE Transactions on Neural Networks and Learning Systems 28(1) (2017), 164-176.

DOI: https://doi.org/10.1109/TNNLS.2015.2504724

[7] L. Li, H. Ge and J. Gao, Maximum-minimum-median average MSD-based approach for face recognition, AEU-International Journal of Electronics and Communications 70(7) (2016), 920-927.

DOI: https://doi.org/10.1016/j.aeue.2016.04.007

[8] S. Su, H. Ge and Y. H. Yuan, A label embedding kernel method for multi-view canonical correlation analysis, Multimedia Tools and Applications 76(12) (2017), 13785-13803.

DOI: https://doi.org/10.1007/s11042-016-3786-3 
[9] S. Su, H. Ge and Y. Tong, Multi-graph embedding discriminative correlation feature learning for image recognition, Signal Processing: Image Communication 60 (2018), 173-182.

DOI: https://doi.org/10.1016/j.image.2017.10.005

[10] H. K. Ji, Q. S. Sun, Y. H. Yuan and Ze-Xuan Ji, C ${ }^{2}$ DMCP: View-consistent collaborative discriminative multiset correlation projection for data representation, Journal of Visual Communication and Image Representation 40(Part B) (2016), 393-405.

DOI: https://doi.org/10.1016/j.jvcir.2016.06.012 